\title{
Laparoscopic Mesogastrium Excision for Gastric Cancer: Only the Beginning
}

\author{
Paolo Ossola, MD, Federico Mascioli, MD, ${ }^{1}$ Diego Coletta, MD, ${ }^{2}$ and Marco Bononi, MD ${ }^{1}$
}

\begin{abstract}
Background: Surgery, with the aid of chemotherapy and radiotherapy, is the only curative chance for gastric cancer. Unfortunately, gastric cancer had an elevated recurrence rate, primarily locally. Mesogastrium excision (MGE) during D2 lymphadenectomy has the aim to remove all possible contaminated tissue around the stomach.

Methods: PubMed, EMBASE, and the Web of Science (WOS) were systematically searched for MGE reports in gastric cancer up to March 2020. The outcome reported were the number of lymph nodes retrieved, operative time (OT), overall morbidity, intra- and postoperative complications, conversion rate, and length of hospital stay.

Results: A total of six studies, including 518 patients, were considered eligible for this analysis. All the studies reported laparoscopic cases. The mean number of lymph nodes retrieved was $36.7 \pm 10.1$. Mean OT was $240.7 \pm 10.1$ minutes. One case of conversion is reported. Overall morbidity was $6 \%$. Medium estimated blood loss was $50.2 \pm 39.6 \mathrm{~mL}$. Overall length of stay was $10.7 \pm 0.7$ days. Mean follow-up was $11 \pm 1.4$ months.

Conclusions: Only few studies evaluated this item, and according to the available evidence, MGE is a feasible technique that could be performed, also laparoscopically, in all surgical resections for gastric cancer with curative intent. Further studies are essential to establish the clear indication of this invasive procedure.
\end{abstract}

Keywords: mesogastric excision, gastric cancer, lymphadenectomy, laparoscopy

\section{Introduction}

G ASTRIC CANCER IS a common gastrointestinal neoplasia, representing the third cause of death for cancer worldwide. ${ }^{1}$ Epidemiological diffusion shows a high prevalence in Asian countries, with $42.6 \%$ of all cases reported in China, ${ }^{1}$ and differences in epidemiological, histopathological, tumor location, diagnostic, and treatment strategy are evident between East and West countries. ${ }^{1}$

Common risk factors for gastric cancer developments are represented by helicobacter pylori infection, diet rich in salt, cigarette smoke, and genetic susceptibility.

When resettable, the only curative treatment option is gastric resection with lymphadenectomy, being chemotherapy and radiotherapy useful only to improve overall survival.

As known, local recurrence is the main reason for death after surgery. Therefore, it is thought to improve local lymphadenectomy associated with mesenterial tissue removal, as already used in mesocolon or mesorectal excision, perform- ing the mesogastrium excision (MGE). Early reports and small case series on this topic seem to give promising outcomes, but the absence of standardization in surgical techniques and the lack of clear evidence preclude its use routinely. The application of this approach during laparoscopic gastric resections represents a further challenge. As in colorectal surgery the development of total mesorectal excision and complete mesocolic excision (CME) identify the gold standard in the surgical treatment of colorectal cancer, this study want to open the debate for a standardization of gastric lymphadenectomy and mesogastric excision for gastric cancer reviewing the available evidences.

\section{Materials and Methods}

We performed a systematic review of published articles according to Preferred Reporting Items for Systematic reviews and Meta-Analyses (PRISMA) guidelines ${ }^{2}$ to identify all studies dealing with lymphadenectomy with mesogastric

\footnotetext{
${ }^{1}$ Pietro Valdoni-Department of Surgery, Umberto I University Hospital, Sapienza University of Rome, Rome, Italy.

${ }^{2}$ Division of Emergency and Trauma Surgery, Department of General Surgery, Emergency Department, Umberto I University Hospital, Sapienza University of Rome, Rome, Italy.
} 
excision during laparoscopic surgery for gastric cancer. PubMed, Embase, Central, and Web of Science electronic databases ${ }^{3}$ were consulted using a combination of the following search words: "mesogastric excision" or "total mesogastric excision" or "mesogastrium." All the authors performed an independent literature screening up to March 2020.

All articles regarding the mesogastric excision during surgery for gastric cancer were considered appropriate. Fulltext articles considered for inclusion were appraised and the relative references were searched to find further eligible articles. All types of articles in English language involving adult patients and about surgery performed in an elective setting entered the analysis. Articles that analyzed only pathological specimens were thus excluded.

Operative outcomes are identified as type of gastrectomy (total or partial), the type of lymphadenectomy (D1 or D2), mean operative time (OT, expressed in minutes), the medium estimated blood loss (EBL, expressed in $\mathrm{mL}$ ), the rate of conversion from laparoscopic to laparotomy procedures, and the rate of reintervention.

Postoperative outcomes are reported as postoperative complications (PC) rate, the length of stay (LOS, expressed in days), the length of the follow-up (expressed in months), and the potential recurrences.

\section{Statistical analysis}

We presented data in descriptive statistics.

Our data were analyzed with Microsoft Excel (Microsoft 365 ) and the SPSS v20.00 software were used when needed (SPSS, Inc., Chicago, IL, USA). All results are expressed as the mean \pm standard deviation, or as frequencies. Differences with $\alpha$-level of $<0.05$ were considered statistically significant.

\section{Results}

\section{Studies selection and quality assessment}

The first database analysis provided 133 results. After the evaluation of abstracts, full texts, and references, six studies, including a total of 518 patients, met the inclusion criteria and were considered in this study (Table 1).

\section{Characteristics of studies}

$\mathrm{All}^{4-9}$ studies were from Asia, in particular four from China, ${ }^{4-7}$ and two from Japan. ${ }^{89}$ Three studies were video reports, ${ }^{4,6}$ and three ${ }^{5,7,8}$ reported the initial results of gastrectomy with MGE. The indication for surgery in all studies was the elective treatment of gastric cancer.
The MGE is named "complete mesogastrium excision" in three studies, ${ }^{4-6}$ "systematic mesogastric excision" in two studies, ${ }^{8,9}$ and in one study "perigastric mesogastrium excision." 7

Data regarding patients' gender are reported in four studies $^{5-8}: 310(67.7 \%)$ men and $148(32.3 \%)$ women are enrolled. Age was indicated in four studies ${ }^{5-8}$ representative for 458 patients, medium age ranged between 55 and 66 years, with medium value of $61.6( \pm 4.7)$ years. Details on body mass index (BMI) were available for 413 patients from three studies, ${ }^{6-8}$ and mean BMI is $22.7( \pm 0.4) \mathrm{kg} / \mathrm{m}^{2}$, varying between 22.3 and $23 \mathrm{~kg} / \mathrm{m}^{2}$.

Operative findings. All surgical procedures were laparoscopic total or partial gastrectomy associated with $\mathrm{D} 1^{8}$ or D2 ${ }^{4-7,9}$ lymphadenectomy and MGE. Mean OT was 240.7 $( \pm 68.9)$ minutes ranging from 127.8 and 303 minutes; OT data resulted in five studies. ${ }^{4-8}$ Five studies ${ }^{4-8}$ indicated medium EBL in 50.2 ( \pm 39.6) $\mathrm{mL}$ (range 12.4-107.1 mL). Only $^{5}$ one case of intraoperative bleeding was detected that led to conversion in laparotomic procedure. No cases of reintervention were noted.

The total number of lymph nodes retrieved were presented in five studies ${ }^{4-8}$ for a total of 512 patients: the mean node collected has been 36.7 ( \pm 10.1$)$ ranging from 31 and 65 .

Postoperative findings. PC were reported in four studies $^{4-7}$ and in 31 cases with a $10.9 \%$ rate (Table 2 summarized). LOS after surgery was also assessed $10.7 \pm 0.7$ days (data from four studies). ${ }^{4-7}$ Two studies ${ }^{6,7}$ reported in $11 \pm 1.4$ months the mean follow-up; Zheng et al. ${ }^{7}$ indicated an hepatic metastasis developed after 11 months from surgery (Table 2).

\section{Discussion}

Surgery is crucial in the treatment of gastric cancer, and in most cases represents the only curative treatment option.

En bloc removal of primary neoplasia associated with D2 lymphadenectomy is considered, in the current International guidelines, the goal in gastric cancer surgical and oncological therapy. ${ }^{1,10-13}$

Unfortunately local recurrence rate in advanced stage of gastric cancer is about $75 \%-80 \%$ after 2 years from gastrectomy with D2 lymphadenectomy ${ }^{14}$ : the reasons of this evidence has to be searched in the prevalence of neoplastic cells, or nodules, in the mesenterial tissue around the stomach that are not removed during standard surgery. This situation is named the "fifth route" of metastasis dispersal, after the

Table 1. Characteristics of Study Selected

\begin{tabular}{llccccc}
\hline $\mathrm{N}$ & \multicolumn{1}{c}{ Refs. } & $M, \mathrm{n}$ & $F, \mathrm{n}$ & Tot, $\mathrm{n}$ & Age, years $(S D)$ & Node, $\mathrm{n}(\mathrm{SD})$ \\
\hline 1 & Xie et al. $^{4}{ }^{5}$ & - & - & 54 & - & $35(10.7)$ \\
2 & Shen et al. & 29 & 16 & 45 & $62(12.3)$ & $35.3(13.1)$ \\
3 & Cao et al. $^{6}$ & 67 & 40 & 107 & $55(3.4)$ & $31(4.6)$ \\
4 & Kumamoto et al. $^{8}$ & 151 & 76 & 227 & $66\left(9.5^{\mathrm{a}}\right)$ & $54\left(24.2^{\mathrm{a}}\right)$ \\
5 & Zheng et al. $^{7}$ & 63 & 16 & 79 & $63.2(9.3)$ & $28.1(10.8)$ \\
6 & Kumamoto et al. $^{9}$ & - & - & 6 & - & - \\
\hline
\end{tabular}

${ }^{a} \mathrm{SD}$ calculated from minimum and maximum range. ${ }^{18}$

$\mathrm{SD}$, standard deviation. 
Table 2. Operative Findings

\begin{tabular}{lc}
\hline OT, mean \pm SD & $240.7 \pm 10.1$ minutes \\
EBL, mean \pm SD & $50.2 \pm 39.6 \mathrm{~mL}$ \\
IO complication, $n(\%)$ & $1(0.2)$ \\
PO complication, $n(\%)$ & $31(10.9)$ \\
Pulmonary infection & $18(58.1)$ \\
Abdominal infection & $1(3.2)$ \\
Anastomotic bleeding & $1(3.2)$ \\
Anastomotic leak & $1(3.2)$ \\
Intestinal obstruction & $2(6.5)$ \\
Deep venous thrombosis & $1(3.2)$ \\
Gastric stasis & $1(3.2)$ \\
Acute kidney failure & $1(3.2)$ \\
Postoperative delirium & $2(6.5)$ \\
Paroxysmal atrial fibrillation & $2(6.5)$ \\
Chilous fistula & $1(3.2)$ \\
Conversion, $n(\%)$ & $1(0.2)$ \\
LOS, mean \pm SD & $10.7 \pm 0.7$ days \\
Follow up, mean \pm SD & $11 \pm 1.4$ months \\
\hline
\end{tabular}

EBL, estimated blood loss; IO, intraoperative; OT, operative time; PO, postoperative.

direct, lymphatic, hematogenic, and peritoneal dissemination. ${ }^{14} \mathrm{~A}$ possible explanation is that all gastric surface is surrounded by a fascia, embryonary residual of gastric mesentery, except from a small area: when neoplasm deeply invaded the gastric wall in this zone, some neoplastic cells seems to fall in the gastric mesenteries. ${ }^{14}$ From these considerations, and in the same manner then for mesorectal and mesocolic excision, was born the idea to remove the mesenterial tissues around the stomach, reducing the chance of neoplastic local recurrence: this procedure was named MGE.

In gastric surgery is not possible to perform a complete excision of the mesenteries as, instead, occurs in colorectal surgery with the mesocolon and mesorectum: the pancreas and the celiac trunk, in fact, must be preserved.

The indications ${ }^{11}$ to complete the D2 lymphadenectomy with MGE are strict: the dissection of mesenteric root (station $14 \mathrm{v}$ ) is suggested in stage III with cancer located in the middle-lower part of stomach or when are individuated metastatic lymph nodes; splenic dissection extension is recommended when cancer dimension $>6 \mathrm{~cm}$, in T3-T4 cancer, when is involved the greater curvature and the middle-upper section of the stomach.

International guidelines ${ }^{1,11-14}$ on gastric cancer reported the minimal number of removal lymph nodes as 16 , and the optimal up to 25. In our series, we reported a mean of $36.7( \pm 10.1)$ lymph nodes retrieved, largely superior to the optimal number recommended: this evidence seems to be due to the surgeon's skills and expertise. MGE, in fact, does not augment the number of lymph nodes retrieved respect D2 lymphadenectomy, but rather the concept to remove lymph nodes and the surrounding adipose tissue as an intact package.

However, even the great number of lymph nodes retrieved with MGE, results on overall and long-term survival in patients undergoing MGE are not yet reported in literature, and the mean follow-up of $11 \pm 1.4$ months is too short.

We observed an intraoperative complications rate of $0.2 \%$, with one case of intraoperative bleeding: we thought that intraoperative vascular lesions during surgery are due to the need of sharp vascular dissection, and are the main cause of

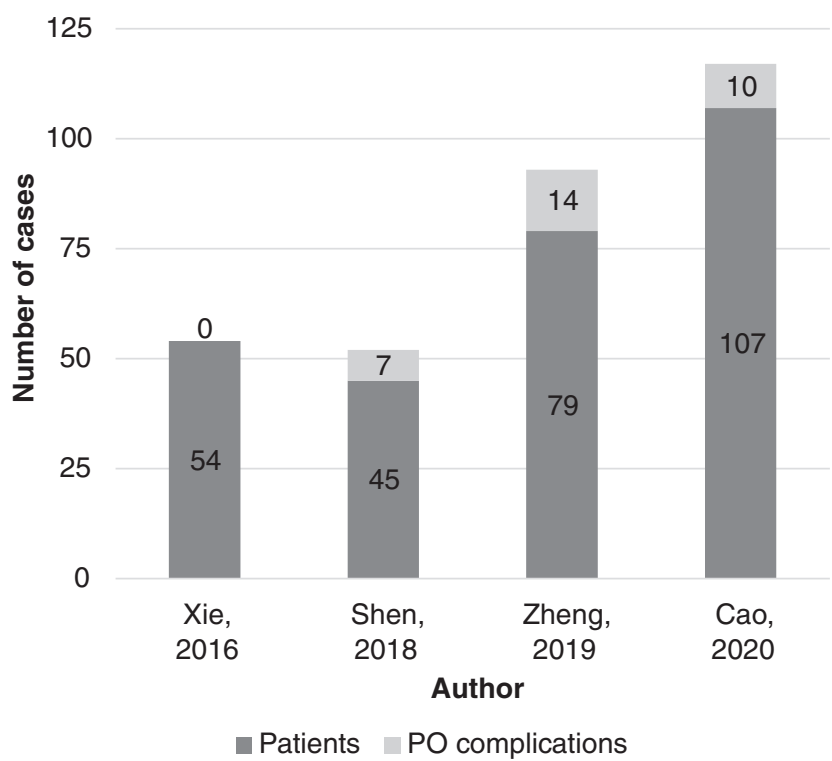

FIG. 1. Postoperative complications.

conversion from laparoscopic to open procedures. Furthermore, we believed that the magnification of the images of a restricted operative field, during these laparoscopy procedures, is a valid help to reduce iatrogenic lesions, and permits a better identification of the embryonary layers. In this sense laparoscopy approach could play a crucial role in the execution of a radical excision. ${ }^{15,16}$

The PC rate was $10.9 \%$ (Fig. 1), being pulmonary infection the most common complication (Table 2). Pulmonary complications represent one of the most frequent causes of postoperative morbidity during upper gastrointestinal surgery with a range of $1.1 \%$ and $12.3 \% .{ }^{17}$ The reasons of this findings are principally due to diaphragmatic irritation during lymphadenectomy, to the protracted inhibition of respiratory and cough centers during anesthesia, and to the limited diaphragmatic mobility for the postoperative pain. ${ }^{17,18}$ Furthermore, the removal of lower esophageal sphincter during total gastrectomy allows the translocation of intestinal material in the esophagus, increasing the risk of aspiration. ${ }^{19}$

Some limitations exist in our study: no data from randomized clinical trials on this topic are available yet; results reported came only from Asian population; a little number of studies are nowadays available.

\section{Conclusions}

In summary, international guidelines ${ }^{1,11-14}$ for gastric cancer treatment recommended standard D2 lymphadenectomy. However, in case of high risk of local recurrence, or when the involvement of distal lymph nodes is suspected, an MGE is proposed, also laparoscopically, to improve the outcomes.

MGE is a feasible technique that could be performed in all surgical resections for gastric cancer with curative intent. The superiority over standard lymphadenectomy in terms of overall survival is still unclear. Further studies, better if comparative, are necessary to define this lymphadenectomy technique as a gold standard in surgical oncology. 


\section{Acknowledgment}

We thank Enrico Ossola for the precious advice and suggestion during the revision of the article.

\section{Authors' Contributions}

Study conception and design, acquisition of data, critical revision of article, and approbation of the final version of article by all authors. Analysis and interpretation of data by D.C. and P.O. Drafting of article by P.O. and F.M.

\section{Disclosure Statement}

No competing financial interests exist.

\section{Funding Information}

No funding was received for this article.

\section{References}

1. Wang FH, Shen L, Li J, et al. The Chinese Society of Clinical Oncology (CSCO): Clinical guidelines for the diagnosis and treatment of gastric cancer. Cancer Commun (Lond) 2019;39:10.

2. Liberati A, Altman DG, Tetzlaff J, Mulrow C, Gøtzsche PC, Ioannidis JP, Clarke M, Devereaux PJ, Kleijnen J, Moher D. The PRISMA statement for reporting systematic reviews and meta-analyses of studies that evaluate healthcare interventions: Explanation and elaboration. BMJ 2009; 339:b2700.

3. Goossen K, Tenckhoff S, Probst P, Grummich K, Mihaljevic AL, Büchler MW, Diener MK. Optimal literature search for systematic reviews in surgery. Langenbecks Arch Surg 2018;403:119-129.

4. Xie D, Yu C, Liu L. Short-term outcomes of laparoscopic D2 lymphadenectomy with complete mesogastrium excision for advanced gastric cancer. Surg Endosc 2016;30: 5138-5139.

5. Shen J, Dong X, Liu Z. Modularized laparoscopic regional en bloc mesogastrium excision (rEME) based on membrane anatomy for distal gastric cancer. Surg Endosc 2018;32: 4698-4705.

6. Cao B, Xiao A, Shen J. An optimal surgical approach for suprapancreatic area dissection in laparoscopic D2 gastrectomy with complete mesogastric excision. J Gastrointest Surg 2020;24:916-917.

7. Zheng CY, Dong ZY, Qiu XT. Laparoscopic perigastric mesogastrium excision technique for radical total gastrectomy. Wideochir Inne Tech Maloinwazyjne 2019;14:229-236.

8. Kumamoto T, Kurahashi Y, Haruta S. Laparoscopic modified lymphadenectomy in gastric cancer surgery using systematic mesogastric excision: A novel technique based on a concept. Langenbecks Arch Surg 2019;404:369-374.

9. Kumamoto T, Kurahashi Y, Niwa H. Laparoscopic suprapancreatic lymph node dissection using a systematic mesogastric excision concept for gastric cancer. Ann Surg Oncol 2020;27:529-531.

10. Waddell T, Verheij M, Allum W, Cunningham D, Cervantes A, Arnold D. Gastric cancer: ESMO-ESSOESTRO clinical practice guidelines for diagnosis, treatment and follow-up. Ann Oncol 2013;24(Suppl. 6):vi57-vi63.

11. Japanese Gastric Cancer Association. Japanese gastric cancer treatment guidelines 2014 (ver. 4). Gastric Cancer 2017;20:1-19.

12. De Manzoni G, Marrelli D, Baiocchi GL. The Italian Research Group for Gastric Cancer (GIRCG) guidelines for gastric cancer staging and treatment: 2015. Gastric Cancer 2017;20:20-30.

13. Zaanan A, Bouché O, Benhaim L. Gastric cancer: French intergroup clinical practice guidelines for diagnosis, treatments and follow-up (SNFGE, FFCD, GERCOR, UNICANCER, SFCD, SFED, SFRO). Dig Liver Dis 2018; 50:768-779.

14. Xie D, Osaiweran H, Liu L. Mesogastrium: A fifth route of metastasis in gastric cancer? Med Hypotheses 2013;80: 498-500.

15. Ossola P, Mascioli F, Coletta D. Laparoscopic and robotic surgery for splenic artery aneurysm: A systematic review. Ann Vasc Surg 2020: S0890-5096(20)30444-1. [Epub ahead of print]; DOI: 10.1016/j.avsg.2020.05.037.

16. Coletta D, Mascioli F, Balla A, Guerra F, Ossola P. Minilaparoscopic cholecystectomy versus conventional laparoscopic cholecystectomy. An endless debate. J Laparoendosc Adv Surg Tech A 2020 [Epub ahead of print]; DOI: $10.1089 /$ lap.2020.0416.

17. Tu RH, Lin JX, Li P, et al. Prognostic significance of postoperative pneumonia after curative resection for patients with gastric cancer. Cancer Med 2017;6:2757-2765.

18. Ntutumu R, Liu H, Zhen L, et al. Risk factors for pulmonary complications following laparoscopic gastrectomy: A single-center study. Medicine (Baltimore) 2016;95:e4567.

19. Shibata $C$, Ogawa H, Nakano $T$, et al. Influence of age on postoperative complications especially pneumonia after gastrectomy for gastric cancer. BMC Surg 2019;19:106.

20. Hozo SP, Djulbegovic B, Hozo I. Estimating the mean and variance from the median, range, and the size of a sample. BMC Med Res Methodol 2005;5:13.

Address correspondence to:

Paolo Ossola, MD

Department of General Surgery

Umberto I University Hospital

Sapienza University of Rome

Viale del Policlinico 155

Rome 00183

Italy

E-mail: paolo.ossola@uniroma1.it 